\title{
Two to tango
}

The death of a cell by apoptosis can occur by either the intrinsic or the extrinsic pathway. Cellular, or more specifically, mitochondrial, stress that is caused by factors such as DNA damage and heat shock triggers the intrinsic pathway. This leads to permeabilization of the outer mitochondrial membrane, a crucial 'point of no return' in the death pathway, which is enabled by pro-apoptotic proteins in the cytoplasm, such as BAX. The release of cytochrome $c$ into the cytosol follows, which promotes the assembly of the apoptosome (a caspase-activating complex), activating downstream caspase proteases; death of the cell ensues. Whereas the role of the

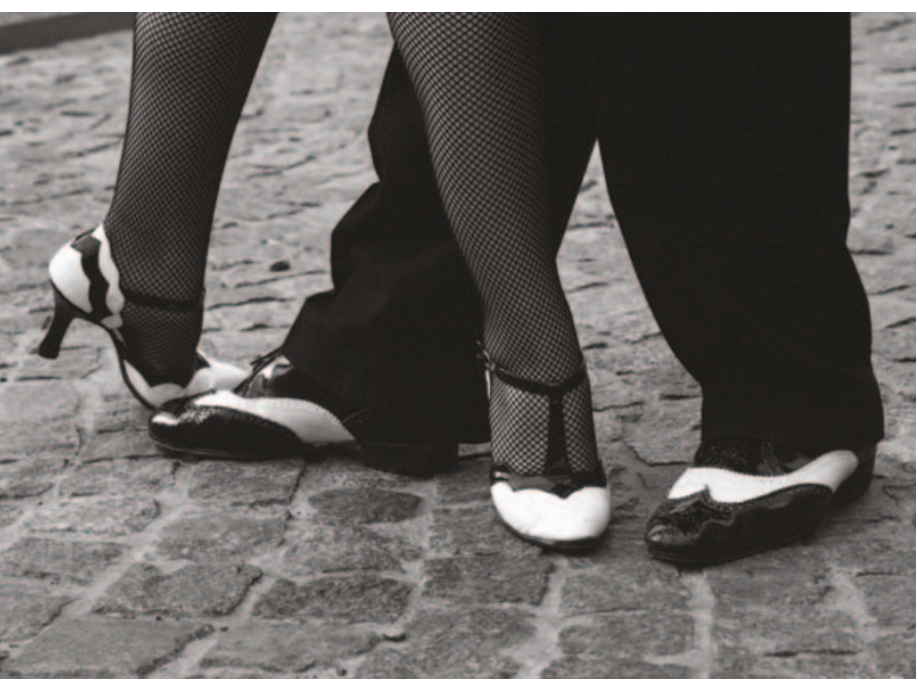

mitochondrion in apoptosis is fairly well defined, Richard Flavell's group, reporting in Science, investigated the functions of caspases in the mitochondrial events of apoptosis. They showed that caspases- 3 and -7 are essential mediators in these processes.

Flavell and colleagues generated mice that lacked the highly related caspases-3 and -7. Mice that lacked one or the other caspase were viable; mice that lacked both caspases- 3 and -7 together, however, had fatal defects in heart development and died rapidly after birth.

So, what roles might caspases- 3 and -7 have together in apoptosis? Experiments in mouse embryonic fibroblasts (MEFs) showed both overlapping and distinct roles: caspase- 3 controlled DNA fragmentation and the morphological changes that are associated with apoptosis, but had a joint role with caspase-7 in the loss of cellular viability, and both were key mediators of the mitochondrial membrane potential. In addition, the release of apoptosisinducing factor, an intramitochondrial protein that is often thought to function in a caspase-independent manner, was shown to be dependent on the combined action of caspases- 3 and -7 .

Because it is thought that mitochondria in cells that are undergoing stress-induced apoptosis might function as amplifiers of caspase activity, the authors asked whether caspases themselves could amplify the mitochondrial events of apoptosis. After exposure to ultraviolet radiation, early apoptotic events in caspase- $3^{-/-}$caspase- $7^{-/-}$double-knockout MEFs, as well as in caspase- $9^{-/-}$MEFs, were delayed. This indicates that there might be a feedback amplification loop - from caspase- 9 through caspases- 3 and -7 - that acts on the initial death signal by promoting the translocation of BAX to the mitochondria and the release of cytochrome $c$ at early time points. The authors noted, however, that these events did occur at later time points.

We are well informed about how caspases are activated during apoptosis, but Flavell and co-workers have now provided much-needed insight into the consequences of the actions of two of these caspases. Although both caspases- 3 and -7 are known to be important in the intrinsic apoptosis pathway, we now know that it is their combined action that is crucial — in this case, it does indeed take two to tango.

Sharon Ahmad

ORIGINAL RESEARCH PAPER Lakhani, S. A. et al. Caspases 3 and 7: key mediators of mitochondrial events of apoptosis. Science $\mathbf{3 1 1}$, 847-851 (2006)

FURTHER READING Adrain, C. \& Martin, S. J. Double knockout blow for caspases. Science 311 785-786 (2006) 